\title{
BM] Global Health Electronic information systems for One Health surveillance of antimicrobial resistance: a systematic scoping review
}

\author{
Madalene Oberin, ${ }^{1,2}$ Skye Badger, ${ }^{1}$ Céline Faverjon, ${ }^{3}$ Angus Cameron, ${ }^{3}$ \\ Melanie Bannister-Tyrrell ${ }^{1}$
}

To cite: Oberin M, Badger S, Faverjon C, et al. Electronic information systems for One Health surveillance of antimicrobial resistance: a systematic scoping review. BMJ Global Health 2022;7:e007388. doi:10.1136/ bmjgh-2021-007388

Handling editor Soumitra S Bhuyan

- Additional supplemental material is published online only. To view, please visit the journal online (http://dx.doi.org/10. 1136/bmjgh-2021-007388).

Received 7 September 2021 Accepted 24 November 2021

A) Check for updates

(c) Author(s) (or their employer(s)) 2022. Re-use permitted under CC BY-NC. No commercial re-use. See rights and permissions. Published by BMJ.

${ }^{1}$ Ausvet, Fremantle, Western Australia, Australia

${ }^{2}$ Faculty of Veterinary and Agricultural Sciences, University of Melbourne, Melbourne, Victoria, Australia

${ }^{3}$ Ausvet Europe, Lyon, France

Correspondence to

Madalene Oberin;

madalene.oberin@ausvet. com.au

\section{ABSTRACT}

Introduction Electronic information systems (EIS)

that implement a 'One Health' approach by integrating antimicrobial resistance (AMR) data across the human, animal and environmental health sectors, have been identified as a global priority. However, evidence on the availability, technical capacities and effectiveness of such EIS is scarce.

Methods Through a qualitative synthesis of evidence, this systematic scoping review aims to: identify EIS for AMR surveillance that operate across human, animal and environmental health sectors; describe their technical characteristics and capabilities; and assess whether there is evidence for the effectiveness of the various EIS for AMR surveillance. Studies and reports between 1 January 2000 and 21 July 2021 from peer-reviewed and grey literature in the English language were included.

Results 26 studies and reports were included in the final review, of which 27 EIS were described. None of the EIS integrated AMR data in a One Health approach across all three sectors. While there was a lack of evidence of thorough evaluations of the effectiveness of the identified EIS, several surveillance system effectiveness indicators were reported for most EIS. Standardised reporting of the effectiveness of EIS is recommended for future publications. The capabilities of the EIS varied in their technical design features, in terms of usability, data display tools and desired outputs. ElS that included interactive features, and geospatial maps are increasingly relevant for future trends in AMR data analytics.

Conclusion No EIS for AMR surveillance was identified that was designed to integrate a broad range of AMR data from humans, animals and the environment, representing a major gap in global efforts to implement One Health approaches to address AMR.

\section{INTRODUCTION}

Electronic information systems (EIS) that aim to capture, analyse and report data about antimicrobial resistance (AMR) in people, animals and the environment are central to the global response to AMR. ${ }^{1}$ International efforts to standardise the collection, analysis, interpretation and sharing of AMR data have been led by the WHO through the Global

\section{Key questions}

What is already known?

- There are numerous global health initiatives aimed to reduce antimicrobial resistance (AMR) prevalence, which encourage implementation of a One Health approach to tackle AMR across the human, animal and environmental health sectors.

- There is limited description and evaluation of electronic information systems (EIS) designed to support One Health surveillance of AMR, especially in lowincome and middle-income countries where the risks posed by AMR are substantial.

\section{What are the new findings?}

- There are no published studies which report comprehensive EIS for AMR surveillance incorporating the entire One Health approach (human, animal and the environmental health sectors).

- There has been limited evaluation of how existing EIS support AMR surveillance.

\section{What do the new findings imply?}

- The development of an EIS which incorporates a One Health approach to AMR surveillance and data remains an important goal that is yet to be achieved.

- Existing EIS for AMR surveillance should be evaluated to determine their effectiveness and potential areas for improvement.

Action Plan on Antimicrobial Resistance (GAP) and the Global Antimicrobial Resistance and Use Surveillance System (GLASS). ${ }^{2}$ Given the spread of resistant bacteria and genes among people, animals, food and the environment, there is global consensus that a collaborative 'One Health' approach should be adopted to respond to AMR. ${ }^{3}$

In 2017, the WHO Advisory Group on Integrated Surveillance of Antimicrobial Resistance (AGISAR) published guidelines for applying a One Health approach to AMR surveillance. ${ }^{4}$ Additionally, WHO, in collaboration with the Food and Agriculture Organisation (FAO) and the World Organisation for Animal Health (OIE), has developed the 
'AMR Tripartite Workplan' in 2019. This plan aims to limit the emergence of AMR by supporting countries to implement a multisectoral One Health approach to AMR through their National Action Plans (NAPs) on AMR. ${ }^{5}$

These initiatives rely on good quality AMR data from humans, animals and the environment to enable integrated surveillance nationally and globally. However, of the 136 countries reporting to the Global Database for the Tripartite Antimicrobial Resistance Country Self-assessment Survey (TrACSS) in 2019-2020, only 32 (24\%) countries include integrated multisectoral AMR surveillance in their NAPs. ${ }^{6}$ Reasons for not having integrated multisectoral surveillance in NAPs may include limited resources, training, knowledge of AMR monitoring initiatives, slow progression of implementation and reduced political authority or willingness to implement strategies. ${ }^{7-10}$

EIS that support AMR surveillance includes a range of online and mobile application-based tools that capture, manage, analyse and report AMR data. These EIS are usually multifaceted and can include a laboratory information management system (LIMS) component to exchange clinical, microbiological, pharmacological and administrative data between laboratories. ${ }^{11}$ For example, 'WHONET', developed by WHO in 1989, is a freely available LIMS that manages microbiology laboratory data and monitors antimicrobial susceptibility in the human health sector. ${ }^{12}$ WHONET is currently used by 130 countries to strengthen their overall AMR surveillance system. However, WHONET is not designed to capture antimicrobial use (AMU) or antimicrobial consumption (AMC) data at the population level. Indeed, for people and animals, AMU and AMC data is predominantly collated in MS Excel spreadsheet templates and submitted to GLASS, WHO or OIE for international reporting. ${ }^{13-15}$ In addition to these limitations, WHONET is not designed to integrate AMR data from the animal and environmental health sectors, though adaptation for animal health is planned. ${ }^{16}$

A systematic review from 2017 examining infectious disease surveillance systems identified a limited number of EIS for AMR. ${ }^{17}$ Importantly, the study highlighted the lack of information systems operating in low-income and middleincome countries (LMICs) where they are most needed because of the high burden of AMR in their populations. ${ }^{18}$ Furthermore, the study objectives for the systematic review by Rattanaumpawan et $a l^{17}$ were limited to the evaluation of EIS in the human health sector only, and EIS with a One Health approach (ie, incorporating human, animal and environmental health data) were not included. Since 2015, many global AMR initiatives have emerged, including the GAP, GLASS, the AMR Tripartite Workplan, AGISAR integrated AMR surveillance guidelines and TrACSS. These initiatives have increased the demand for EIS tailored to One Health AMR data. To the authors knowledge, no recently published study of such EIS exists, leaving the current global landscape of EIS for integrated AMR surveillance in humans, animals and the environment uncharted.
Our scoping review aims to address this gap. Specifically, we aim to (1) identify EIS that collect, integrate and analyse AMR surveillance data across all health sectors (ie, human, animal and the environment) that have been designed, piloted and/or implemented in the past 5years, (2) describe the technical characteristics and capabilities of the identified EIS, (3) establish whether an evaluation of the effectiveness of the identified EIS has been conducted, consistent with the US Centers for Disease Control and Prevention (CDC) guidelines and (4) provide recommendations for the future development of EIS to facilitate AMR data sharing and integration across sectors.

\section{METHODS}

This study was designed as a systematic scoping review with a qualitative synthesis of evidence to identify current knowledge gaps and investigate the literature available using a systematic search process. Conducting and reporting of results were in accordance with the Preferred Reporting Items for Systematic Reviews and Meta-Analyses (PRISMA) guidelines for Scoping Reviews (PRISMA-ScR). ${ }^{19}$

\section{Eligibility criteria}

This review defined an EIS for AMR as any online or mobile application-based tool that integrates, manages, analyses and reports on AMR data. For this review, data on AMU or AMC were considered 'AMR data'. An EIS for AMR surveillance could be part of a local, regional, national or international surveillance system. We included studies and technical reports published in the English language between 1 January 2000 and 21 July 2021 that described any form of an EIS that was (1) currently operational, being piloted or under investigation, (2) intended to assist with the monitoring or surveillance of AMR and (3) covered at least one of the three health sectors (human, animal or environment). Studies were excluded if they were unrelated to an EIS for AMR data or did not describe the design or implementation of the EIS.

\section{Information sources}

PubMed Central and Web of Science electronic databases were the primary information source for this review. Grey literature was explored to enhance the electronic database search by scanning the reference list of included articles, and reviewing WHO, FAO and OIE websites related to AMR. Specifically, the NAPs of the countries which are included in the WHO library of $\mathrm{NAPs}^{20}$ were screened for the key search terms to determine whether there was sufficient description of any EIS for AMR surveillance to meet the eligibility criteria for this review. Google Scholar was used as a secondary database to scan for additional studies using the same search terms as the primary search (table 1 ).

\section{Search}

The sources and terms used are denoted in table 1.

\section{Selection of sources of evidence}

Search results from each source were exported into the Zotero referencing software for the identification, merging 


\begin{tabular}{|c|c|c|}
\hline Source & Search terms & Search dates \\
\hline PubMed & $\begin{array}{l}\text { (“2000/01/01”(Date - Publication): “2021/07/21”(Date - Publication)) AND (surveillance(Title/ } \\
\text { Abstract)) AND ((electronic(Title/Abstract)) OR (digital(Title/Abstract)) OR (automated(Title/ } \\
\text { Abstract)) OR (real-time(Title/Abstract)) OR (real time(Title/Abstract))) AND (antimicrobial } \\
\text { resistan*(Title/Abstract)) }\end{array}$ & $\begin{array}{l}1 \text { January } 2000 \text { to } 21 \\
\text { July } 2021\end{array}$ \\
\hline Web of Science & $\begin{array}{l}(\mathrm{AB}=(\text { surveillance } \mathrm{AND} \text { (electronic OR digital OR automated OR real-time OR real time) AND } \\
\left.\text { antimicrobial resistan })^{*}\right)\end{array}$ & $\begin{array}{l}1 \text { January } 2000 \text { to } 21 \\
\text { July } 2021\end{array}$ \\
\hline Google Scholar & $\begin{array}{l}\text { (Antimicrobial resistance surveillance) AND ((electronic) OR (digital) OR (automated) OR (real-time) } \\
\text { OR (real time)) }\end{array}$ & $\begin{array}{l}1 \text { January } 2000 \text { to } 21 \\
\text { July } 2021\end{array}$ \\
\hline Grey Literature & $\begin{array}{l}\text { The NAPs of countries recorded within the WHO library of national action plans (NAPs) were } \\
\text { extracted and assessed to identify any electronic information systems (EIS) or potentially } \\
\text { developing EIS that met the inclusion criteria, further described in online supplemental table } \\
\text { S4. Other organisations (such as Food and Agriculture Organisation and World Organisation for } \\
\text { Animal Health), and the reference list of included studies were also scanned. }\end{array}$ & 2000 to $2021^{*}$ \\
\hline
\end{tabular}

*The earliest NAP included in this study was published in 2011.

and removal of duplicates. Screening of retrieved titles and abstracts was performed in Zotero by two reviewers independently. Disagreements were resolved by mutual agreement. Full texts were accessed to assess eligibility and decide on inclusion.

\section{Data items and charting processes}

Data items were extracted and entered in a MS Excel spreadsheet, and the specific domains are described in table 2. For each domain, a variety of data items were extracted. First, the study characteristics were identified by the country economic status in which each EIS was deployed, the phase of development, health sectors involved and the population source used to retrieve the data. Second, for the technical and design characteristics, the usability, interactivity, security, structure and storage capabilities of each EIS were identified. Lastly, the surveillance systems effectiveness evaluation tool referred to as the 'Centers for Disease Control (CDC) Updated Guidelines for Evaluating Public Health Surveillance Systems, ${ }^{21}$ was used to identify whether indicators of surveillance system effectiveness were reported for the EIS included in this review. The CDC guidelines describe indicators in terms of relevant attributes such as stability, representativeness, timeliness, simplicity, acceptability, flexibility and data quality, to evaluate surveillance systems, and have previously been applied to evaluate AMR surveillance systems. ${ }^{22}$

\section{RESULTS \\ Selection of sources of evidence}

A total of 547 records were assessed, comprising 466 records from the two primary databases, 3 records from Google Scholar and 78 records from the grey literature (figure 1). After duplicate records were removed ( $\mathrm{n}=158)$, a total of 389 records were screened, of which 345 were excluded at the title and abstract screening stage or the key terms screening stage in the NAPs, leaving 44 records for full-text screening. Of these, 18 records were excluded because they did not meet the inclusion criteria, leaving 26 studies and reports for inclusion in the review. In relation to the grey literature, five of the eight included studies that had been peer reviewed and the remaining three studies were sourced from government reports.

\section{Characteristics of sources of evidence}

Twenty-two of the 26 studies and reports described unique EIS for AMR, and 4 studies described implementations of WHONET. ${ }^{1223-25}$ One study included the analysis of 2 EIS, ${ }^{26}$

Table 2 Data collection domains for data extraction and charting

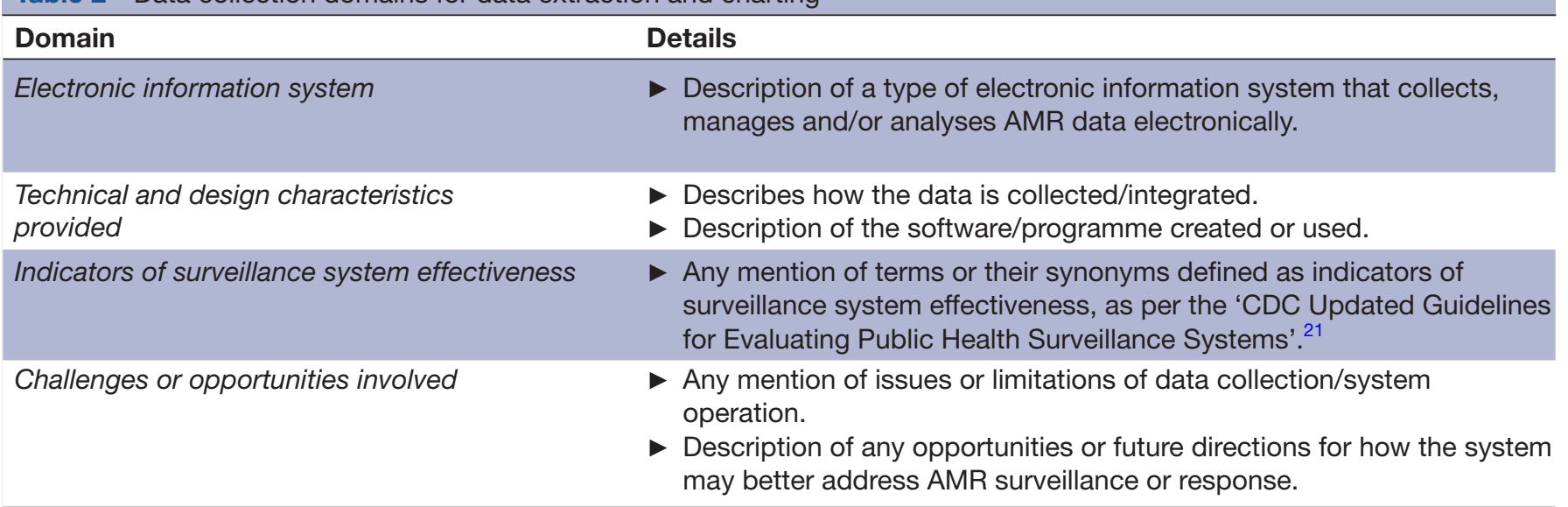

AMR, antimicrobial resistance; CDC, Centers for Disease Control and Prevention. 


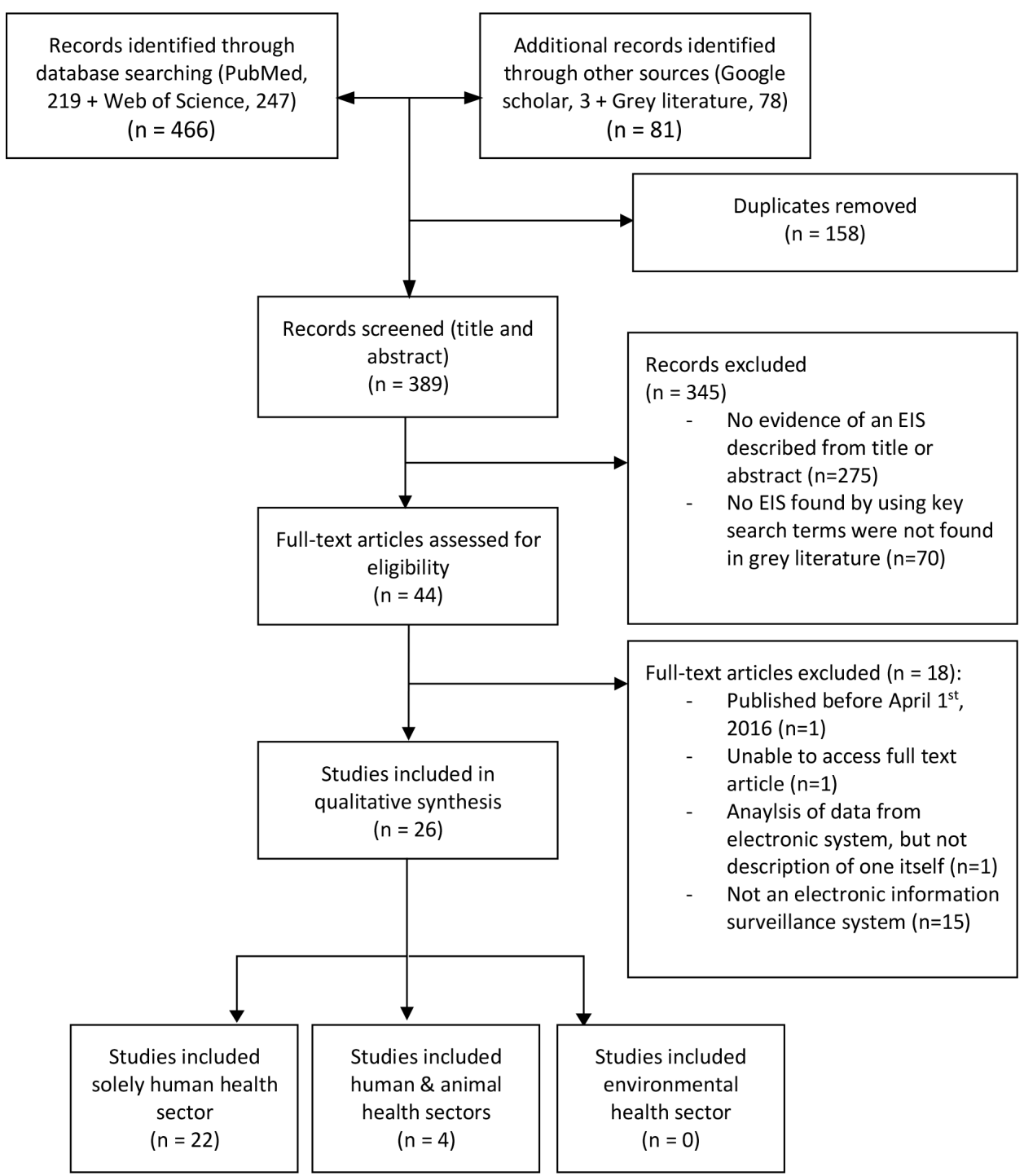

Figure 1 Study selection. Preferred Reporting Items for Systematic Reviews and Meta-Analyses 2020 flowchart for selection of studies and reports in the systematic scoping review. EIS, electronic information systems.

resulting in a total of 27 EIS overall included in this systematic scoping review from 26 studies and reports. Of the 27 EIS, the country of implementation was specified for 21: Uganda, ${ }^{27}$ India, ${ }^{28}$ Portugal, ${ }^{29}$ Italy, ${ }^{23}$ Australia,${ }^{30-32}$ Netherlands, ${ }^{33}$ Germany, ${ }^{34}$ Brazil, ${ }^{35}$ UK, ${ }^{36}$ USA, ${ }^{24}{ }^{37}{ }^{38}$ Argentina, ${ }^{25}$ Taiwan, ${ }^{39}$ France, ${ }^{26}$ Russia, ${ }^{40}$ Switzerland ${ }^{41}$ and Denmark. ${ }^{42}$ Three EIS described utilisation across multiple countries, ${ }^{12434}$ and three EIS are not specific to a location. ${ }^{45-47}$

\section{Synthesis of results}

Objective 1: identify EIS that collect, integrate and analyse AMR surveillance data across health sectors (human, animal and the environment)

The EIS were described using various characteristics including (1) the economic status of the country of implementation, (2) the phase of the EIS, (3) the health sectors involved and (4) the population sources from which the data is collected. The characteristics of the 27 EIS evaluated are described in table 3 (additional information on each EIS can be found in online supplemental table S1). The four reports that use WHONET, ${ }^{12} 23-25$ involved one describing the use solely within a hospital setting, ${ }^{23}$ and the other three operated within both hospital and community settings. ${ }^{12}$ One of the WHONET systems reported integrated data from public health, veterinary and food laboratories, but did not include the environmental health sector. ${ }^{12}$ One EIS did not distinguish between human or hospital settings (unspecified) but collected metagenomic sequencing data, which was described in terms of its public health relevance. ${ }^{47}$ None of the 27 EIS evaluated in this review reported the inclusion of AMR data from environmental sources (such as wastewater, agricultural runoff, groundwater, rivers or other sources) ${ }^{48}$

Objective 2: describe the technical characteristics and capabilities of the identified EIS

The technical characteristics and capabilities of the EIS were assessed on design features including (1) user accessibility and usability, (2) information visualisation and interactivity, (3) database security and confidentiality, (4) database structure and (5) storage. The features of each EIS are described 


\begin{tabular}{|c|c|}
\hline EIS characteristics & Number of EIS (n=27) $n(\%)$ \\
\hline \multicolumn{2}{|l|}{$\begin{array}{l}\text { Country economic } \\
\text { status }^{*}\end{array}$} \\
\hline Low-income & $1(3.7)^{27}$ \\
\hline $\begin{array}{l}\text { Low-income and } \\
\text { middle-income }\end{array}$ & $1(3.7)^{28}$ \\
\hline Middle-income & $2(7.4)^{2535}$ \\
\hline High-income & $17(63)^{23242629-3436-3840-4249}$ \\
\hline Not specific & $6(22)^{1243-47}$ \\
\hline Total & 27 \\
\hline \multicolumn{2}{|l|}{ Phase of EIS } \\
\hline Operational & $22(81.5)^{122426-2931-3740-4244-4749}$ \\
\hline Piloted & $4(14.8)^{23253843}$ \\
\hline Under construction & $1(3.7)^{30}$ \\
\hline Total & 27 \\
\hline \multicolumn{2}{|l|}{ Health sector/s } \\
\hline Human (only) & $23(81)^{23-36} 384042-44464749$ \\
\hline Human and animal & $4(19)^{12374145}$ \\
\hline Environment & 0 \\
\hline Total & 27 \\
\hline \multicolumn{2}{|l|}{ Population source ${ }^{\dagger \ddagger \S}$} \\
\hline \multicolumn{2}{|l|}{ Human (only) } \\
\hline Hospital & $11(33.3)^{23262729343538434449}$ \\
\hline $\begin{array}{l}\text { Hospital and } \\
\text { community }\end{array}$ & $11(42.9)^{24252830-3336404246}$ \\
\hline Unspecified & $1(4.8)^{47}$ \\
\hline \multicolumn{2}{|l|}{ Human and animal } \\
\hline $\begin{array}{l}\text { Hospital and retail } \\
\text { meats/slaughter }\end{array}$ & $1(4.8)^{37}$ \\
\hline $\begin{array}{l}\text { Hospital, } \\
\text { community } \\
\text { and veterinary } \\
\text { laboratory }\end{array}$ & $3(14.3)^{124145}$ \\
\hline Total & 27 \\
\hline
\end{tabular}

${ }^{*}$ Country economic status categorised by the World Bank. ${ }^{69}$ †Hospital data: AMR data collected from people who have been hospitalised.

$\ddagger$ Community data: Data collected from people who are in the community, such as samples taken or requested by general practitioners, or from outpatients.

$\S$ The population source contains EIS that collect data from humans and animals, which have been broken down into subcategories for clarity.

AMR, antimicrobial resistance; EIS, electronic information systems.

in (table 4, online supplemental table S2 describes the characteristics of each EIS). Some of the EIS lacked sufficient published information to determine all their technical characteristics and capabilities. While usability and accessibility vary across the EIS, all are accessible freely and easily online. Many of the systems require users to create a profile and enter login information to upload and access the data. Additional

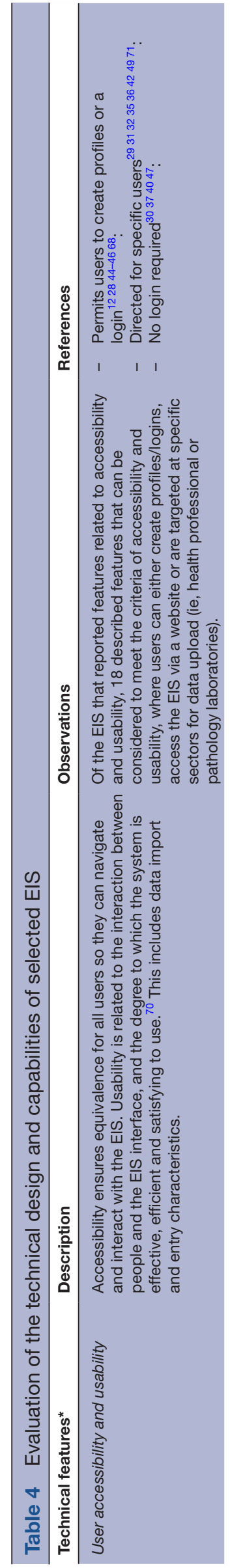


features for users were identified across the EIS, for example, the ResistanceOpen EIS describe features that allow users to set up data elements, data entry forms and reports, as well as being compatible on a variety of devices. ${ }^{46}$ The data output and display of all EIS consisted of a form of an analysis report, however additional interactivity was observed in several EIS which allows for various visual representations of the data. ${ }^{27} 30$ 35-37 404649 For example, HOTSpots and ResistanceOpen allows users to use navigable maps, which indicates the geographical location and saturation of the data at various levels with different intensity and layers. ${ }^{3046}$ The security features of the EIS mostly involve password-protected accounts for users and de-identified data. The backend structure of EIS evaluated describe specific programming language to perform queries and analysis the data to produce the output results. They also involve storing the data within a cloud, web-based server or on a local server, of which both consist of various security and safety properties.

Objective 3: identify whether indicators of surveillance system effectiveness have been applied to evaluate AMR electronic information systems

The reviewed EIS varied in reporting whether the contribution of the EIS to AMR surveillance system effectiveness had been formally assessed using indicators of surveillance system effectiveness, including stability, representativeness, timeliness, simplicity, acceptability, flexibility, data quality, positive predictive value and sensitivity. Many of the included studies and reports explicitly described at least one indicator, most commonly representativeness, though for most indicators, there were only limited indirect reference to the indicator without clear evidence for whether this indicator had been formally evaluated (table 5). For example, some reports described EIS as 'user friendly', which was considered an indirect reference to the indicator 'simplicity'. Further information is provided in the supplementary materials (online supplemental table S3).

\section{DISCUSSION}

\section{Summary of evidence}

Our review of currently available EIS for AMR surveillance did not identify any system that adopted a multisectoral One Health approach that integrated data across the human, animal and environmental health sectors. While a limited number of reviewed EIS incorporated human and animal health data, none of the reviewed systems explicitly included AMR in the environment.

Table 5 Extent to which indicators of surveillance system effectiveness were applied to included EIS

\begin{tabular}{|c|c|c|c|c|}
\hline \multirow{2}{*}{$\begin{array}{l}\text { Surveillance system } \\
\text { effectiveness indicators }\end{array}$} & \multirow[b]{2}{*}{ Definition } & \multicolumn{3}{|c|}{ Observed for the EIS $(n=27)$} \\
\hline & & Yes* & Not & Indirectlył \\
\hline \multirow[t]{2}{*}{ Stability } & Refers to the reliability and availability EIS. & $\begin{array}{l}\mathrm{n}=2 \\
4449\end{array}$ & $\begin{array}{l}\mathrm{n}=4 \\
263132\end{array}$ & $\begin{array}{l}n=21 \\
1223-25 \text { 27-30 33-38 40-43 }\end{array}$ \\
\hline & & & & $45-47$ \\
\hline Representativeness & $\begin{array}{l}\text { Accurately describes the occurrence of } \\
\text { a health-related event (ie, AMR) and its }\end{array}$ & 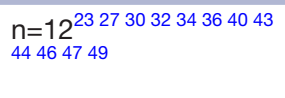 & $\begin{array}{l}n=1 \\
45\end{array}$ & $\begin{array}{l}\mathrm{n}=14^{12} 24-262829313335 \\
37384142\end{array}$ \\
\hline
\end{tabular}
distribution in the population by place and person.

\begin{tabular}{|c|c|c|c|c|}
\hline Timeliness & $\begin{array}{l}\text { The timely process of an EIS to present the } \\
\text { data once collected. }\end{array}$ & $\mathrm{n}=7^{25273334424449}$ & $\begin{array}{l}\mathrm{n}=4 \\
3136-38\end{array}$ & $\begin{array}{l}\mathrm{n}=16^{12} 23242628293233 \\
3540414345-47\end{array}$ \\
\hline Simplicity & $\begin{array}{l}\text { Clear and functionality of the EIS in terms of its } \\
\text { structure and ease of operation. }\end{array}$ & $n=3^{344346}$ & $\begin{array}{l}\mathrm{n}=6 \\
2631323638\end{array}$ & $\begin{array}{l}\mathrm{n}=18 \\
1223-2527-3033353740-42 \\
44454749\end{array}$ \\
\hline Acceptability & $\begin{array}{l}\text { Willingness of persons/organisations to } \\
\text { participate/contribute data for the EIS. }\end{array}$ & $n=2^{2743}$ & $\begin{array}{l}\mathrm{n}=7 \\
2630-323638\end{array}$ & $\begin{array}{l}n=18^{12} 23-25282933-3537 \\
40-4244-4749\end{array}$ \\
\hline Flexibility & $\begin{array}{l}\text { The ability to adapt to changing information } \\
\text { needs or operating conditions with little } \\
\text { additional time, personnel or allocated funds. }\end{array}$ & $\mathrm{n}=5^{1234374249}$ & $\begin{array}{l}\mathrm{n}=11 \\
24-2630-33363840\end{array}$ & $\begin{array}{l}\mathrm{n}=11 \\
2327-29354143-47\end{array}$ \\
\hline Data quality & $\begin{array}{l}\text { Reflects the completeness and validity of the } \\
\text { data recorded in the public health surveillance } \\
\text { system. }\end{array}$ & $n=61227334349$ & $\begin{array}{l}n=7 \\
24-26313238\end{array}$ & $\begin{array}{l}n=14 \\
2328-3034-3740-42444547\end{array}$ \\
\hline Positive predictive value & $\begin{array}{l}\text { The proportion of clusters or outbreaks } \\
\text { detected by the EIS that were confirmed to } \\
\text { be clusters or outbreaks after epidemiological } \\
\text { investigation. }\end{array}$ & $n=5^{24263849}$ & $\begin{array}{l}\mathrm{n}=222^{122327-37} \\
40-47\end{array}$ & $\mathrm{n}=0$ \\
\hline
\end{tabular}

*Yes=the study or report described how the EIS had been evaluated for this indicator, or provided substantial description of how this indicator was incorporated.

†No=there was no mention of the indicator, or no evidence of it being explicitly described.

fIndirectly=there was enough sufficient evidence to infer that the indicator was described, although not enough information to determine whether the indicator was analysed or evaluated.

AMR, antimicrobial resistance; EIS, electronic information systems. 
This finding highlights a significant gap in our response to the global threat posed by AMR. The omission of environmental AMR data in EIS is of concern given groups such as the United Nations Environment Assembly and the Interagency Coordination Group on AMR have highlighted a critical need to further understand the role of environmental pollution in the emergence and spread of $\mathrm{AMR}^{50} 51$

In this study, the 21 EIS that identified a specific country of implementation, were mostly located in HICs $(n=17)$, with a smaller number implemented in LMICs $(n=4)$. Additionally, two of the six EIS which included a range of countries were found to involve predominantly LMICs (such as Southeast Asian countries), ${ }^{43} 44$ demonstrating a slight overall increase in the presence of EIS in LMICs over the past 5 years since a previous review in $2017^{17}$ of a similar scope. The growing number of EIS being deployed in LMICs in recent years may be due to the heightened awareness at national and global levels about AMR, increased access to digital health technologies in LMICs, availability of international funding initiatives for implementing EIS as components of AMR surveillance or increased demand for AMR data in recent years. ${ }^{7852}$ However, the slow progress in the development and adoption of EIS for AMR surveillance in LMICs likely involves many contributory factors, such as being heavily dependent on grants, or awaiting national or international agencies' support. ${ }^{7}$

WHONET appears to be the principal EIS tool used for AMR data in most countries. ${ }^{12}$ However, within our systematic search, just four studies described the implementation of WHONET, of which two were implemented in the past 5 years, ${ }^{123}$ and the other two in the past 11 years. ${ }^{24} 25$ Two were pilot studies describing its implementation in Italy and Argentina, ${ }^{23} 25$ and one other was operational in Boston (USA), ${ }^{24}$ all three were used to compare the EIS to the current operational surveillance networks within the selected hospitals. The fourth study was a descriptive summary of WHONET. ${ }^{12}$ Given the longstanding and widespread use of WHONET, this constitutes a clear gap in the documentation of implementation experiences, its added value to countries' health systems and in identifying strengths and weaknesses to improve the system further.

When identifying whether evaluations of the EIS had been conducted using the CDC guidelines of surveillance system effectiveness, we found that majority of the reports did not assess how the EIS contributed to the effectiveness of AMR surveillance overall. Of the 27 EIS, representativeness was described most frequently $(n=12)$, as EIS commonly described the source and target population for the AMR data, and how it related to the occurrence and distribution of AMR. However, the other indicators were typically described or discussed indirectly, rather than an explicit evaluation of how the EIS improved AMR surveillance (table 5). A recent study of AMR surveillance systems ${ }^{22}$ highlighted that not all desirable attributes described in the CDC guidelines can necessarily be implemented together in a single system. It was recommended that a priority list of indicators for the most 'effective' AMR surveillance systems be developed. ${ }^{22}$ However, for this to be useful, studies and reports on EIS for AMR should provide more data on the contribution of EIS to AMR surveillance system effectiveness overall. The lack of high-quality evaluation data may impede future investment opportunities in developing integrated EIS for AMR surveillance at the national and international levels.

Our grey literature search showed that although many countries did not indicate the deployment of an EIS (or report the existence of an EIS), some countries indicate within their NAPS that they are planning to implement such a system in the future (online supplemental table S4). This is a positive indication that increased attention and resources are being dedicated to improving the availability of AMR data through EIS. In particular, the OIE noted from a 2020 survey that there is a lack of an electronic tool that is available to monitor AMU in animals that can collect and analyse data. ${ }^{53}$ Hence, they reported that they are now in the process of developing an interactive automated system to fill this gap in reporting AMU in the animal health sector.

From the NAPs investigated in this review (online supplemental table S4), it was evident that there is a lack of information about existing EIS. For example, little reference was made to EIS in the NAPs of Japan or Sweden even though these countries have established EIS. ${ }^{54}{ }^{55}$ While overall, we found limited evidence of integrated EIS, some national surveillance programmes such as NORM-VET, EARS-VET, DANMAP, CIPARS and RESAPTH include AMR data from both humans and animals, ${ }^{56-60}$ indicating features of a One Health approach. Evidently, the various EIS described within this review retrieved from the published and grey literature records provides an overview of what is currently available or being developed for AMR. However, it was challenging to find reliable information to guide further developments or identify the characteristics of these EIS as not all countries share this information publicly.

While we found a paucity of publicly available information on private or government sponsored EIS for AMR and AMU data, it is not inferred that data collection tools do not exist in the human and animal sectors, particularly in high-income countries. For example, Varona et al report on several databases used to managed AMR and AMU data in six European countries. ${ }^{61}$ Problems were identified with a lack of harmonisation and standardisation in data types and reporting within and across sectors. Further, few of the databases were considered capable of a truly One Health approach to AMR and AMU surveillance. ${ }^{61}$ One EIS of interest, the Electronic Communication of Surveillance in Scotland, was found to have insufficient publicly available information to include in this review. ${ }^{61}$ 


\section{Recommendations}

A possible strategy to overcome the gap in knowledge on the performance of EIS is to develop a set of standardised requirements to be reported within published papers describing the development, implementation and evaluation of EIS. These guidelines would be similar to those published for the reporting of other scientific studies, such as 'PRISMA' for reporting systematic reviews, or Strengthening the Reporting of Observational Studies in Epidemiology guidelines for the reporting of observational studies, and the Equator Network which strives for enhancing the quality and transparency of health research. ${ }^{62-64}$ Additionally, reporting on EIS in GLASS, TrACSS or OIE surveys may also help to provide muchneeded information on the existence and performance of EIS for AMR surveillance at national level.

Several evaluation tools exist for the surveillance of AMU and AMR in addition to the CDC guidelines discussed in this review, such as 'ATLASS: the Assessment Tool for Laboratories and AMR Surveillance Systems', and 'ISSEP: Integrated Surveillance System Evaluation Project' ${ }^{65}$ We recommend such platforms become more standardised in terms of the attributes described, and to be additionally integrated into the rollout of WHONET and other EIS to improve the performance assessments of these systems in the future. There is an opportunity for an EIS to be developed like the universal applicability of WHONET, which also incorporates a wider range of AMR data by capturing AMU and AMC data, as well as enabling a One Health approach by integrating data from human, animal and environmental health sectors. A system with an interactive, responsive feed of real-time AMR data targeted for all stakeholders from all sectors would be valuable for national and global AMR responses.

The COVID-19 pandemic has accelerated technological trends occurring in EIS and highlighted the critical role of data analytics in public health. ${ }^{66}$ Accessibility, usability and collaboration are critical to achieving EIS data integration across multiple health sectors. Among the digital technologies predicted in the short-term to medium-term, the greatest disrupters are considered to be automation, artificial intelligence and 5G connectivity. ${ }^{667}$ EIS need to be designed with flexibility and scalability to accommodate these and other emerging trends in the future. The EIS examined in this study all demonstrated an aspect of recording and reporting AMR data, however the EIS that incorporated additional features such as being able to function offline, ${ }^{27}{ }^{44}$ incorporate interactivity, processing real-time results and geospatial mapping features may be considered more aligned with future innovations, ${ }^{3035-37404668}$ and therefore being more effective than those that lack those characteristics. The rapid pace of technological advancements demands EIS that are flexible and responsive to digital disruption. Therefore, EIS with the capacity to upscale and adapt to new technologies are needed to remain relevant for AMR surveillance. For example, cloud based servers are increasingly popular due to their efficiency in providing readily available and scalable solutions and cost efficiencies compared with on-site, local servers with large data centres requiring additional overhead costs and problems in recovering lost data. ${ }^{67}$ The EIS that describe deployment on local servers may consider adapting or updating their database to align with digital advances. Additionally, to incorporate a One Health approach, many of the EIS evaluated in this review may need to adapt to meet the global desire to integrate data across human and animal health, and the environment.

There is a distinct lack of publicly available information regarding EIS developments; the Tripartite or similar initiative should consider establishing a global repository of EIS for AMR. Governments, international organisations or the private sector could submit a brief description of the status and planned developments of an EIS to ensure there is information available, and to more easily identify or evaluate the effectiveness of the various information systems and data management practices being used.

\section{Limitations}

Methodological limitations of this review include that we did not search for pre-print literature, thereby potentially missing very recent information. Second, we only searched among literature in English language. Third, our search strategy for grey literature did not follow a traditional systematic approach. Instead, the WHO library of $\mathrm{NAPs}^{20}$ was the main grey literature resource, as well as the reference lists of studies, and other relevant government and health organisation resources were reviewed selectively based on prior knowledge among the researchers. This may have resulted in some publication bias in the results reported in this review, where not all EIS currently in use were able to be evaluated. Further, it was challenging to properly assess whether indicators of surveillance system effectiveness had been applied, as most studies did not provide sufficient detail on the technical capabilities or user features or critique the performance of the systems. We recommend that information related to the design and overall effectiveness of EIS for AMR surveillance be broadly shared to encourage the collaborative development of systems that integrate multisectoral data.

Surveillance of environmental AMR is not well established in many countries. Therefore, true integration of One Health data across all sectors (human, animals and the environment) is not yet possible in many countries. While the EIS included in this study do not incorporate environmental AMR data, some systems may be able to accommodate this data (eg, WHONET). Certainly, in the future, EIS for integrated One Health data should be designed to accommodate AMR data from all sectors.

\section{CONCLUSION}

This scoping review maps the current landscape of EIS for AMR surveillance. There remains a clear need for EIS that truly implement a One Health approach to capture the contribution of all health sectors towards the emergence of 
AMR. The gaps identified in this review provide important guidance to improve current and develop future systems. Additionally, for countries, organisations or researchers planning and developing an EIS for AMR, or aiming to strengthen current systems, this review highlights the importance of researching and/or evaluating how EIS strengthens AMR surveillance systems overall.

Contributors $\mathrm{MO}$ is the author acting as guarantor of this paper. M0, SB, CF, AC and MB-T contributed to the conception, plan and design of the study. MO and MB$\mathrm{T}$ collected the data and performed the analysis. M0 and MB-T wrote the first draft of the manuscript, contributions from SB and CF were included in later drafts. All authors contributed by reading, revising and approving the submitted version.

Funding This research was partly supported through a Fleming Fund Country Grant to Indonesia (2019-2021).

Competing interests None, all authors are employed by the Ausvet Group via its companies Ausvet and Ausvet Europe.

Patient consent for publication Not applicable.

Ethics approval This study does not involve human participants. The research involved publicly available, anonymised information only. No ethics approval to conduct the study was obtained or sought for this research.

Provenance and peer review Not commissioned; externally peer reviewed.

Data availability statement Data are available in a public, open access repository. All data relevant to the study are included in the article or uploaded as supplementary information.

Supplemental material This content has been supplied by the author(s). It has not been vetted by BMJ Publishing Group Limited (BMJ) and may not have been peer-reviewed. Any opinions or recommendations discussed are solely those of the author(s) and are not endorsed by BMJ. BMJ disclaims all liability and responsibility arising from any reliance placed on the content. Where the content includes any translated material, BMJ does not warrant the accuracy and reliability of the translations (including but not limited to local regulations, clinical guidelines, terminology, drug names and drug dosages), and is not responsible for any error and/or omissions arising from translation and adaptation or otherwise.

Open access This is an open access article distributed in accordance with the Creative Commons Attribution Non Commercial (CC BY-NC 4.0) license, which permits others to distribute, remix, adapt, build upon this work non-commercially, and license their derivative works on different terms, provided the original work is properly cited, appropriate credit is given, any changes made indicated, and the use is non-commercial. See: http://creativecommons.org/licenses/by-nc/4.0/.

\section{REFERENCES}

1 World Health Organization. Antimicrobial resistance. Available: https://www.who.int/news-room/fact-sheets/detail/antimicrobialresistance [Accessed 19 Feb 2021].

2 Global antimicrobial resistance and use surveillance system (glass). Available: https://www.who.int/initiatives/glass [Accessed 31 May 2021].

3 United Nations. At un, global leaders commit to act on antimicrobial resistance. Available: https://www.who.int/news/item/21-09-2016at-un-global-leaders-commit-to-act-on-antimicrobial-resistance [Accessed 29 Jul 2021].

4 World Health Organization. Integrated surveillance of antimicrobial resistance in foodborne bacteria: application of a one health approach. Copenhagen: Regional Office for Europe, 2017.

5 Food and Agriculture Organization of the United Nations, World Organisation for Animal Health, World Health Organization. AMR Tripartite Workplan (updated-08-April-2019). Available: http://web. oie.int/downld/WG/AMR/AMR-Tripartite-Workplan-updated-08-April2019.pdf [Accessed 13 Apr 2021].

6 World Health Organization (WHO). Food and Agriculture Organization of the United Nations (FAO) and World Organisation, for Animal Health (OIE) Monitoring global progress on antimicrobial resistance: Tripartite AMR country self-assessment survey (TrACSS) 20192020. Available: https://www.who.int/publications-detail-redirect/ monitoring-global-progress-on-antimicrobial-resistance-tripartiteamr-country-self-assessment-survey-(tracss)-2019-2020 [Accessed July 29, 2021]
7 Iskandar K, Molinier L, Hallit S, et al. Surveillance of antimicrobial resistance in low- and middle-income countries: a scattered picture. Antimicrob Resist Infect Control 2021;10:63.

8 Chua AQ, Verma M, Hsu LY, et al. An analysis of national action plans on antimicrobial resistance in Southeast Asia using a governance framework approach. Lancet Reg Health West Pac 2021;7:100084.

9 Al-Haboubi M, Trathen A, Black N, et al. Views of health care professionals and policy-makers on the use of surveillance data to combat antimicrobial resistance. BMC Public Health 2020;20:279.

10 Joshi MP, Chintu C, Mpundu M, et al. Multidisciplinary and multisectoral coalitions as catalysts for action against antimicrobial resistance: implementation experiences at national and regional levels. Glob Public Health 2018;13:1781-95.

11 Centers for Disease Control and Prevention, Electronic laboratory reporting (ELR). How does ELR work? Available: https://www.cdc. gov/elr/how-does-elr-work.html [Accessed 10 Jun 2021].

12 Stelling J, O'Brien TF. WHONET: software for surveillance of infecting microbes and their resistance to antimicrobial agents. Molecular Microbiology 2016:692-706.

13 World Health Organization. Who methodology for point prevalence survey on antibiotic use in hospitals, 2018. Available: https://www. who.int/publications-detail-redirect/WHO-EMP-IAU-2018.01

14 World Health Organization. Glass manual on the management of antimicrobial consumption data, 2020. Available: https://apps.who. int/iris/handle/10665/336216

15 World Organisation for Animal Health. Guidance for completing the OIE template for the collection of data on antimicrobial agents intended for use in animals. Available: https://www.oie.int/app/ uploads/2021/03/eng-amuse-guidance-final-2020.pdf [Accessed 29 Jul 2021].

16 FAO. FAO Evaluation of FAO's role and work on antimicrobial resistance (AMR. Rome, Italy, 2021.

17 Rattanaumpawan P, Boonyasiri A, Vong S, et al. Systematic review of electronic surveillance of infectious diseases with emphasis on antimicrobial resistance surveillance in resource-limited settings. Am $J$ Infect Control 2018;46:139-46.

18 Turner P, Rupali P, Opintan JA, et al. Laboratory informatics capacity for effective antimicrobial resistance surveillance in resource-limited settings. Lancet Infect Dis 2021;21:e170-4.

19 Tricco AC, Lillie E, Zarin W, et al. PRISMA extension for scoping reviews (PRISMA-ScR): checklist and explanation. Ann Intern Med 2018;169:467-73.

20 World Health Organization. Library of national action plans. Available: https://www.who.int/teams/surveillance-preventioncontrol-AMR/national-action-plan-monitoring-evaluation/library-ofnational-action-plans [Accessed 02 Nov 2021]

21 Centers for Disease Control and Prevention. Updated guidelines for evaluating public health surveillance systems, 2001. Available: https://www.cdc.gov/mmwr/preview/mmwrhtml/rr5013a1.htm [Accessed 10 May 2021].

22 Al-Haboubi M, Glover RE, Eastmure E, et al. Quality and utility of information captured by surveillance systems relevant to antimicrobial resistance (AMR): a systematic review. Antibiotics 2021; $10: 431$.

23 Natale A, Stelling J, Meledandri M, et al. Use of WHONET-SaTScan system for simulated real-time detection of antimicrobial resistance clusters in a hospital in Italy, 2012 to 2014. Euro Surveill 2017;22. doi:10.2807/1560-7917.ES.2017.22.11.30484. [Epub ahead of print: 16 Mar 2017].

24 Huang SS, Yokoe DS, Stelling J, et al. Automated detection of infectious disease outbreaks in hospitals: a retrospective cohort study. PLoS Med 2010;7:e1000238.

25 Stelling J, Yih WK, Galas M, et al. Automated use of WHONET and SaTScan to detect outbreaks of Shigella spp. using antimicrobial resistance phenotypes. Epidemiol Infect 2010;138:873-83.

26 Abat $\mathrm{C}$, Chaudet $\mathrm{H}$, Colson P, et al. Real-time microbiology laboratory surveillance system to detect abnormal events and emerging infections, Marseille, France. Emerg Infect Dis 2015:21:1302-10.

27 Lamorde M, Mpimbaza A, Walwema R, et al. A cross-cutting approach to surveillance and laboratory capacity as a platform to improve health security in Uganda. Health Secur 2018;16:S76-S-86.

28 Kaur J, Sharma A, Dhama AS, et al. Developing a hybrid antimicrobial resistance surveillance system in India: Needs \& challenges. Indian J Med Res 2019;149:299-302.

29 Simões AS, Maia MR, Gregório J, et al. Participatory implementation of an antibiotic stewardship programme supported by an innovative surveillance and clinical decision-support system. J Hosp Infect 2018;100:257-64. 
30 Wozniak TM, Cuningham W, Buchanan S, et al. Geospatial epidemiology of Staphylococcus aureus in a tropical setting: an enabling digital surveillance platform. Sci Rep 2020;10:13169.

31 Australian Commission on Safety and Quality in Health Care. CARAlert annual report: 2019, 2019. Available: https://www. safetyandquality.gov.au/publications-and-resources/resource-library/ caralert-annual-report-2019 [Accessed 19 Apr 2021].

32 Australian Commission on Safety and Quality in Health Care. Australian passive antimicrobial resistance surveillance (APAS) first report: multi-resistant organisms. Available: https://www. safetyandquality.gov.au/publications-and-resources/resource-library/ australian-passive-antimicrobial-resistance-surveillance-apas-firstreport-multi-resistant-organisms [Accessed 19 Apr 2021]

33 Altorf-van der Kuil W, Schoffelen AF, de Greeff SC, et al. National laboratory-based surveillance system for antimicrobial resistance: a successful tool to support the control of antimicrobial resistance in the Netherlands. Euro Surveill 2017;22.

34 Schweickert B, Feig M, Schneider M, et al. Antibiotic consumption in Germany: first data of a newly implemented web-based tool for local and national surveillance. $J$ Antimicrob Chemother 2018;73:3505-15.

35 Pillonetto M, Jordão RTdeS, Andraus GS, et al. The experience of implementing a national antimicrobial resistance surveillance system in Brazil. Front Public Health 2020;8:575536.

36 Johnson AP, Muller-Pebody B, Budd E, et al. Improving feedback of surveillance data on antimicrobial consumption, resistance and stewardship in England: putting the data at your fingertips. $J$ Antimicrob Chemother 2017;72:dkw536.

37 Karp BE, Tate H, Plumblee JR, et al. National antimicrobial resistance monitoring system: two decades of advancing public health through integrated surveillance of antimicrobial resistance. Foodborne Pathog Dis 2017;14:545-57.

38 Graham PL, San Gabriel P, Lutwick S, et al. Validation of a multicenter computer-based surveillance system for hospitalacquired bloodstream infections in neonatal intensive care departments. Am J Infect Control 2004;32:232-4.

39 Abujnah AA, Zorgani A, Sabri MAM, et al. Multidrug resistance and extended-spectrum $\beta$-lactamases genes among Escherichia coli from patients with urinary tract infections in Northwestern Libya. Libyan J Med 2015;10:26412.

40 Kuzmenkov AY, Trushin IV, Vinogradova AG, et al. AMRmap: an interactive web platform for analysis of antimicrobial resistance surveillance data in Russia. Front Microbiol 2021;12:620002.

41 Federal Office of Public Health and Federal Food Safety and Veterinary Office. Swiss antibiotic resistance report 2020. usage of antibiotics and occurrence of antibiotic resistance in Switzerland, 2020. Available: https://www.anresis.ch/wp-content/uploads/ 2020/11/Swiss-Antibiotic-Resistance-Report-2020 def_WEB.pdf [Accessed 11 May 2021].

42 Voldstedlund M, Haarh M, Mølbak K, et al. The Danish microbiology database (MiBa) 2010 to 2013. Euro Surveill 2014;19:20667.

43 Turner P, Ashley EA, Celhay OJ, et al. Acorn (a clinically-oriented antimicrobial resistance surveillance network): a pilot protocol for case based antimicrobial resistance surveillance. Wellcome Open Res 2020;5:13

44 Lim C, Miliya T, Chansamouth V, et al. Automating the generation of antimicrobial resistance surveillance reports: proof-of-concept study involving seven hospitals in seven countries. J Med Internet Res 2020;22:e19762.

45 Thomsen MCF, Ahrenfeldt J, Cisneros JLB, et al. A bacterial analysis platform: an integrated system for analysing bacterial whole genome sequencing data for clinical diagnostics and surveillance. PLOS One 2016;11:e0157718.

46 MacFadden DR, Fisman D, Andre J, et al. A platform for monitoring regional antimicrobial resistance, using online data sources: ResistanceOpen. J Infect Dis 2016;214:S393-8.

47 Lakin SM, Dean C, Noyes NR, et al. MEGARes: an antimicrobial resistance database for high throughput sequencing. Nucleic Acids Res 2017:45:D574-80.

48 Singer AC, Shaw H, Rhodes V, et al. Review of antimicrobial resistance in the environment and its relevance to environmental regulators. Front Microbiol 2016;7:7.

49 Tseng Y-J, Wu J-H, Ping X-O, et al. A web-based multidrugresistant organisms surveillance and outbreak detection system with rule-based classification and clustering. J Med Internet Res 2012;14:e131.

50 Interagency Coordination Group (IACG). No time to wait: securing the future from drug-resistant infections. Available: https://www.who. int/antimicrobial-resistance/interagency-coordination-group/IACG final_report_EN.pdf?ua=1 [Accessed 06 Sept 2021].
51 Gaze W, Depledge M. Antimicrobial resistance: investigating the environmental dimension - frontiers 2017: emerging issues of environmental concern, 2017. Available: https://wedocs.unep.org/ handle/20.500.11822/22263

52 Hoque R, Ahmed SM, Naher N, et al. Tackling antimicrobial resistance in Bangladesh: a scoping review of policy and practice in human, animal and environment sectors. PLoS One 2020;15:e0227947.

53 World Organisation for Animal Health. OIE annual report on antimicrobial agents intended for use in animals. Available: https:// www.oie.int/app/uploads/2021/03/a-fourth-annual-report-amr.pdf [Accessed 22 Oct 2021].

54 Kajihara T, Yahara K, Hirabayashi A, et al. Japan nosocomial infections surveillance (JANIS): current status, international collaboration, and future directions for a comprehensive antimicrobial resistance surveillance system. Jpn J Infect Dis 2021;74:87-96.

55 Söderblom T, Billström H, Kahlmeter G. Working with the Swedish early warning and antimicrobial resistance surveillance system SVEBAR. European Congress of clinical microbiology and infectious diseases. Abstract, 2014. Available: https://www.escmid.org/ escmid_publications/escmid_elibrary/material/?mid=14923

56 NORM-VET reports. Available: https://www.vetinst.no/en/ surveillance-programmes/norm-norm-vet-report [Accessed $22 \mathrm{Apr}$ 2021].

57 Mader R, Damborg P, Amat J-P, et al. Building the European antimicrobial resistance surveillance network in veterinary medicine (EARS-Vet). Eurosurveillance 2021;26:2001359.

58 Resapath French surveillance network for antimicrobial resistance in bacteria from diseased animals. Available: https://www.anses.fr/ fr/system/files/LABO-Ra-Resapath2018EN.pdf [Accessed 12 Apr 2021].

59 DANMAP. Use of antimicrobial agents and occurrence of antimicrobial resistance in bacteria from food animals food and humans in Denmark, 2019. Available: Https://Www.Danmap.Org [Accessed 09 Apr 2021].

60 Canada PHA. Canadian integrated program for antimicrobial resistance surveillance (CIPARS) 2018: Executive summary, 2018. Available: https://www.canada.ca/en/public-health/services/ surveillance/canadian-integrated-program-antimicrobial-resistancesurveillance-cipars/cipars-reports/2018-annual-report-executivesummary.html [Accessed 10 Aug 2021].

61 Mesa Varona O, Chaintarli K, Muller-Pebody B, et al. Monitoring antimicrobial resistance and drug usage in the human and livestock sector and foodborne antimicrobial resistance in six European countries. Infect Drug Resist 2020;13:957-93.

62 von Elm E, Altman DG, Egger M, et al. Strengthening the reporting of observational studies in epidemiology (STROBE) statement: guidelines for reporting observational studies. BMJ 2007;335:806-8.

63 Page MJ, McKenzie JE, Bossuyt PM, et al. The PRISMA 2020 statement: an updated guideline for reporting systematic reviews. PLoS Med 2021;18:e1003583.

64 The UK EQUATOR. The EQUATOR network: enhancing the quality and transparency of health research. Available: https://www.equatornetwork.org/about-us/ [Accessed 29 Jul 2021].

65 Sandberg M, Hesp A, Aenishaenslin C, et al. Assessment of evaluation tools for integrated surveillance of antimicrobial use and resistance based on selected case studies. Front Vet Sci 2021:8:620998

66 Walter C, Walter C. Future trends in geospatial information management: the five to ten year vision. 82. ordnance survey of great Britain, United nations Committee of experts on global Geospatial information management, 2020. https://www.geobusinessshow. com/events/future-trends-in-geospatial-information-managementthe-5-to-10-year-vision/

67 Al-Marsy A, Chaudhary P, Rodger JA. A model for examining challenges and opportunities in use of cloud computing for health information systems. Appl Syst Innov 2021;4:15.

68 DHIS2. District health information System-2, 2021. Available: https:// dhis2.org/ [Accessed 27 Jul 2021].

69 The World Bank. World bank country and lending groups. Available: https://datah elpdesk.worldbank.org/knowledgebase/articles/ 906519-world-bank- country-andlending-groups [Accessed 28 May 2021].

70 International Organization for Standardization. Ergonomics of human-system interaction - part 11: usability: definitions and concepts. Available: https://www.iso.org/obp/ui/\#iso:std:iso:9241:11:ed-2:v1:en [Accessed 05 Aug 2021]

71 Infect - interface for empirical antimicrobial chemotherapy. Available: https://www.infect.info [Accessed $30 \mathrm{Jul}$ 2021]. 